\title{
IMPORTÂNCIA DA APLICAÇÃO DA FERRAMENTA 5S DE QUALIDADE EM UM CENTRO AUTOMOTIVO
}

\author{
Cleber Gomes Pinto Filho \\ Centro Universitário La Salle do Rio de Janeiro \\ https://www.unilasalle.edu.br/rj \\ cleber.filho@soulasalle.com.br \\ Suzana Arleno Souza \\ Centro Universitário La Salle do Rio de Janeiro \\ https://www.unilasalle.edu.br/rj \\ suzana.santos@lasalle.org.br
}

\begin{abstract}
RESUMO
Esta pesquisa tem como objetivo principal ressaltar a importância da ferramenta $5 \mathrm{~s}$ no sistema operacional de um centro automotivo. O 5 s com o auxílio do senso organizacional oferece uma qualidade personalizada a cada tipo de serviço. Com a facilidade de ser obter um veículo automotor nos dias de hoje, o mercado de serviços e produtos automotivos cresceu com grande força de aceitação do público, e para se diferenciar dos demais a qualidade tem sido um fator de extremo peso. O projeto baseia-se em dois tipos de metodologia: a bibliográfica e prática, conhecida também como investigação de campo, tornando-se então um estudo exploratório. Para a coleta dos dados foi realizado um estudo em parceria com o Centro Automotivo NEWCAR, localizado no bairro de Jardim Catarina, São Gonçalo-RJ, com início no mês de maio de 2018 e término em janeiro de 2019 . Oferecer suporte para melhoria e métodos de se manter sempre a frente das concorrentes foram os principais pontos abordados.
\end{abstract}

Palavra-chave: Melhoria contínua; Ferramenta 5s; Qualidade; Garantia de qualidade.

\begin{abstract}
The main objective of this research is to emphasize the importance of the $5 \mathrm{~s}$ tool in the operating system of an automotive center. The $5 \mathrm{~s}$ with the aid of organizational sense offers a personalized quality to each type of service. With the ease of getting a self-propelled vehicle nowadays, the market for automotive services and products has grown with great force of public acceptance, and to differentiate itself from the others the quality has been a factor of extreme weight. The project is based on two types of methodology, the bibliography and practice, also known as field research, thus becoming an exploratory study. For data collection, a study was conducted in partnership with the NEWCAR Automotive Center, located in the neighborhood of Jardim Catarina, São Gonçalo-RJ, beginning in the month of may 2018 and ending in january 2019. Providing support for improvement and methods of always keeping ahead of competitors were the main points addressed.
\end{abstract}

Keywords: Continuous improvement; 5s Tool; Quality; Quality Assurance. 


\section{INTRODUÇÃO}

Melhoria contínua é o verdadeiro sinônimo para manter um constante sucesso dentro

de uma organização. Com o passar dos tempos, os gerentes operacionais começaram a notar que aprimorar seus conhecimentos e manter a disciplina de suas ações acabariam resultando em pontos positivos para a empresa.

Aperfeiçoar os seus produtos, serviços e linha de produção se tornou uma ação aderida por toda empresa que busca se tornar melhor a cada dia que passa. Além de ser um fator crucial, este acaba fortalecendo ainda mais o desempenho diante de seus concorrentes. Seguir a linha de pensamento Toyotista: "Hoje melhor do que ontem, amanhã melhor do que hoje" acabou fazendo sentido para aqueles que buscavam de alguma maneira superar suas metas.

Pode-se afirmar que todo o sucesso por trás desse pensamento vem do planejamento de suas ações. A lógica por baixo de todo o processo torna concreta a ideia de Pedro Carlos de Carvalho (2011), que acreditava que a análise construtiva do empreendimento está relacionada a todas as partes do método de produção e prática construtiva.

Para que todo o sucesso almejado fosse alcançado de forma prática, surgiu-se então, no Japão, no início da década de 50 a ferramenta 5 s de qualidade. Não se pode afirmar quem realmente criou a metodologia, porém muitos autores acreditam que o verdadeiro responsável

pelo surgimento desta foi o Dr. Kaoru Ishikawa (1989), um grande engenheiro de qualidade, $\mathrm{e}$

criador de muitos métodos teóricos para administração japonesa.

Diante do exposto, objetivo principal deste estudo é aumentar a qualidade dos serviços de um centro automotivo. Portanto, viu-se necessário a implementação de uma ferramenta simples e que ofereça bons resultados, e com isso a proposta do $5 \mathrm{~s}$ de diminuir os desperdícios e aumentar a eficiência da operação fez todo sentido permitindo desenvolver a melhoria contínua na destinação dos materiais.

\section{MÉTODOS QUALITATIVOS}

\subsection{QUALIDAde TotaL}

Segundo Geraldo Vieira (2003), Gestão Qualidade Total, na língua Inglesa Total Quality Management ou TQM, é uma especialidade da administração em que, de forma estratégica, orienta todo o seu processo organizacional. O conceito de total é referente ao fato

de envolver todos os seus parceiros no processo, desde de seu fornecedor de matéria prima até

o transportador para realizar, de forma eficaz, as entregas.

Diretamente ligada ao Toyotismo, e superando de forma positiva o fordismo, no

TQM os colaboradores recebem suporte para produzir bens e serviços respondendo concretamente às necessidades dos clientes, aprendem a garantir a sobrevivência da empresa de acordo com um lucro contínuo - resultado do domínio da qualidade - e principalmente a reduzir os desperdícios como também identificar e solucionar os caminhos críticos, por meio de sua prioridade. (Pareto). 


\subsubsection{Metodologia 5s}

O $5 \mathrm{~s}$ é um conjunto de cinco palavras de origens japonesas que começam a letra $\mathrm{S}$, como apresenta a figura 01, com o objetivo de simplificar a melhora dos hábitos dentro de uma empresa, a fim de obter um aumento significativo em seus resultados. Nesta tecnologia a interação com todos os setores é um fator de extrema importância para o sucesso. A prática desta ferramenta proporciona aprendizado e melhora não só a qualidade do serviço como também na vida do profissional dentro da organização.

De acordo com o DR Kaoru Ishikawa (1997), o 5s é visto como uma grande faxina dentro da empresa e com isso é imprescindível que se torne rotina dentro dela. Esta ideia acaba explorando muito bem três extensões, o layout espaço físico para execução do processo,

a intelectual - voltada à realização das tarefas - e a social visando os relacionamentos do diaa-dia. E de acordo com este também, ao modificarmos algum cenário, os outros acabam sendo afetados positivamente.

Para Gracielle Alves e Valério Garcia (2014), mostrar que nada é inatingível e tudo pode ser melhorado fez fortalecer ainda mais os pontos para encorajar as pessoas utilizarem tal metodologia. A semelhança com o pensamento dos japoneses ao desenvolverem o programa $5 \mathrm{~s}$ passou segurança a todos que desejavam fazer o uso desta ferramenta que é representado pelas 5 palavras:

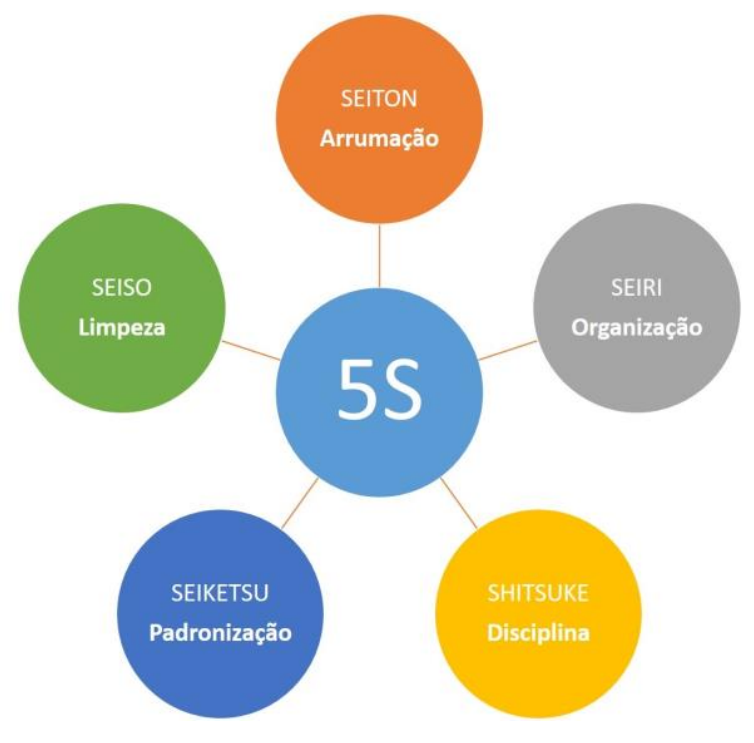

Figura 01 Base conceituando o significado de cada $\mathrm{S}$ Fonte: http://blog.nxfacil.com.br/metodologia-5s/

Portanto, vale ressaltar que o $5 \mathrm{~s}$ aplicado de maneira correta pode melhorar o sistema de valores da empresa, relações sociais e energia para desempenho das tarefas. Porém deve-se atentar aos riscos que esse sistema pode trazer caso não seja realizado de forma correta e principalmente rotineira. Se no senso de utilização os colaboradores não aderirem à ideia, o ambiente ficará sobrecarregado e afetará negativamente todo o resto. Por isso, importante explicar de forma bem clara os motivos pelo qual o $5 \mathrm{~s}$ foi escolhido, fazendo que todos entendam os seus benefícios. 


\subsubsection{A metodologia 5s dentro de um Centro Automotivo}

Oficina mecânica é um ambiente conhecido popularmente por sua extrema desordem e falta de limpeza. Peças de todos os tipos espalhados pelo campo de trabalho acabam fazendo com que tudo fique fora de controle e muitas vezes, por conta da falta de ordem, acabe ocorrendo o desperdício de algum produto e acarrete prejuízos.

Além de ser notoriamente um diferencial, manter uma oficina organizada oferece facilidade na execução de seus trabalhos, estimula os funcionários a trabalharem melhor, acaba com as perdas desnecessárias por falta de controle e deixa o cliente satisfeito. Conquistar a confiança do cliente faz uma extrema diferença neste ramo. Oferecer um serviço de qualidade, além de fidelizar faz com que a fama do estabelecimento se propague, visto que todos que contrataram algum serviço e gostaram irão passar esta informação para frente, como uma espécie de marketing involuntário, e novos possíveis clientes surgirão. Ao oferecer uma estrutura diferenciada das demais, levando em consideração que a maioria das oficinas não prezam pela organização, o contratante irá se sentir acolhido e confiante de que seu carro receberá um serviço de primeira linha, assim como o estabelecimento se encontra.

Com isso, a metodologia $5 \mathrm{~s}$ se tornou uma maneira de trazer organização para dentro deste ambiente. Podendo ser aplicado em todas as áreas de uma oficina, o mesmo não necessita de um investimento alto e por isso se tornou acessível.

O primeiro senso em questão, é conhecido como senso de utilização, o Seiri, tratase do ponto de partida para implantação da metodologia 5s. Para tal é imprescindível que seja separado tudo que é útil e não útil para utilização. Dentro do plano de melhoria, este primeiro passo esteve atrelado à identificação dos produtos que não são constantemente utilizados no dia a dia, mas que também não podem ser descartados e os que definitivamente não possuem utilidade nenhuma e por isso devem ser descartados.

O Seiton, identificado como o senso de ordenação, coloca em ordem materiais, objetos e informações que possam ser úteis de maneira funcional, sendo o seu principal intuito oferecer um rápido acesso ao que se deseja. Para que este próximo passo seja executado de maneira correta dentro da oficina e apresente bons resultados, concluiu-se que deveria ser realizado a conscientização da equipe sobre a importância de se trabalhar em um local onde todos os itens possuem uma ordem. Todos do estabelecimento devem entender a filosofia por trás do 5s. De nada adiantaria arrumar e logo após alguém tirar tudo do lugar e não colocar novamente. A disciplina, palavra chave do último senso, deve estar presente em todas as etapas.

O terceiro senso tem um papel não só de limpar o campo de trabalho, mas também de mantê-lo organizado sempre. Identificar o motivo de tudo ficar tão sujo e eliminá-lo, se tornou o verdadeiro significado do Seiso. Dentro de um centro automotivo a limpeza faz toda a diferença na recepção de seus clientes. Chegar a um local organizado, clean, conquista a confiança de seus clientes e até cultiva uma possível fidelização do mesmo.

O quarto senso está voltado à saúde e higiene local. Zelar por um ambiente onde a saúde física e mental de seus colaboradores seja prioridade da empresa. Oferecer um campo para trabalho, em que todas as ações levem em consideração o que beneficiaria na vida de sua equipe, faz com que todos os envolvimentos sejam estimulados para realizar suas tarefas de forma positiva, ou oferecendo o seu melhor, visto que a empresa se preocupa com todo o seu quadro. Fazendo da vida uma via de mão dupla, em que a empresa zela pela saúde de seus funcionários e estes zelam pela imagem da empresa.

Autodisciplina e o último S, aponta a necessidade das pessoas a se atendarem da importância de todos os sensos anteriores, a fim de manter de forma continua a realização de todas as tarefas. Para que seja uma ordem rotineira e constante, toda a equipe precisa se atentar a sua autodisciplina, com determinação e sempre com vontade de obter um resultado ainda melhor. 
Conhecido também como o fiscal, esta parte do processo oferece toda estrutura necessária para identificar se a ferramenta 5 s estar sendo utilizada de forma correta ou não. Desde o comprometimento de seus colaboradores até real necessidade de utilização algum objeto dentro da oficina.

\subsection{FERRAMENTA 5W2H}

De acordo com o SEBRAE (2008), o 5w2h é classificado como uma ferramenta prática que possibilita que seja encontrado, a qualquer momento, informações e dados que sejam importantes para a produção ou um projeto. O seu conceito deve-se a utilização de 7 palavras da língua inglesa: What (o que, qual), Where (onde), Who (quem), Why (porque, para que), When (quando), How (como) e How Much (quanto, custo). Com esta ferramenta também é possível encontrar o perfil de cada um dos envolvidos no projeto, facilitando na hora de distribuir as tarefas de cada um.

Conforme apresenta na figura 02, o método consiste em responder as sete perguntas, em que todos os aspectos, básicos e essenciais, sejam analisados como forma de orientação de ações que deverão ser utilizadas.

O 5w2h consegue dar uma visão bem objetiva de tudo que acontece no projeto, podendo então prevenir-se de possíveis falhas na execução do trabalho. E com isso, diante de toda problemática dentro de gerenciamento de projetos e informações, essa metodologia, por meio de conclusões básicas, vem oferecendo informações importantes para a contextualização de um projeto.

\begin{tabular}{|c|l|l|l|}
\hline \multirow{3}{*}{$\mathbf{5 W}$} & & \multicolumn{2}{|c|}{ Método dos 5W2H } \\
\cline { 2 - 4 } & What & O Que? & Que ação será executada? \\
\cline { 2 - 4 } & Who & Quem? & Quem irá executar/participar da ação? \\
\cline { 2 - 4 } & Where & Onde? & Onde será executada a ação? \\
\cline { 2 - 4 } & When & Quando? & Quando a ação será executada? \\
\cline { 2 - 4 } & Why & Por Quê? & Por que a ação será executada? \\
\hline \multirow{2}{*}{$\mathbf{2 H}$} & How & Como? & Como será executada a ação? \\
\cline { 2 - 4 } & How much & Quanto custa? & Quanto custa para executa a ação? \\
\hline
\end{tabular}

Figura 02 - Quadro Comparativo entre os métodos $5 \mathrm{~W}$ e $2 \mathrm{H}$

Fonte: SEBRAE (2008)

\section{METODOLOGIA}

Para desenvolvimento do trabalho a abordagem bibliográfica foi mais do que necessária, pois apenas a partir dos fundamentos teóricos foi possível realizar a prática desta metodologia. Porém a pesquisa de campo, de como funciona na prática, também foi colocada em execução, seguindo então o pensamento exposto por Andrade (1993), em que o conteúdo direto refere-se à parte bibliográfica e o indireto à pesquisa de campo, como avalições e visitas ao local.

É denominada também de acordo com Gil (2010) como uma pesquisa exploratória, pois tem o intuito de projetar, entender e desenvolver conceitos e ideias. Este tipo de pesquisa apresenta forte base teórica, a fim de fundamentar todos os argumentos a serem expostos e executados de maneira correta.

Com embasamento nas informações acima, foi criado um fluxograma do processo, como apresenta a figura 03, com as seguintes etapas, analisar a situação atual (a identificação do problema), impactar positivamente a direção (apresentar as vantagens da metodologia), pesquisa pré-implantação (reconhecimento do local para implantação), apresentar aos funcionários/treinamento (explicar a metodologia e como utilizar), organização da 
equipe/divisão de tarefas (selecionar cada perfil e distribuir a equipe de acordo com a identificação com as tarefas), Implantação, semana D (analise dos resultados durante o tempo de utilização), e avalição (estudar os funcionários na utilização dos sensos). $\mathrm{O}$ objetivo era de mapear e facilitar a execução desta ferramenta. Foram identificados os pontos que deveriam ser trabalhados, e com isso, a partir da fundamentação teórica, sugerir possíveis melhorias ao local escolhido - no caso em tela, o centro automotivo.

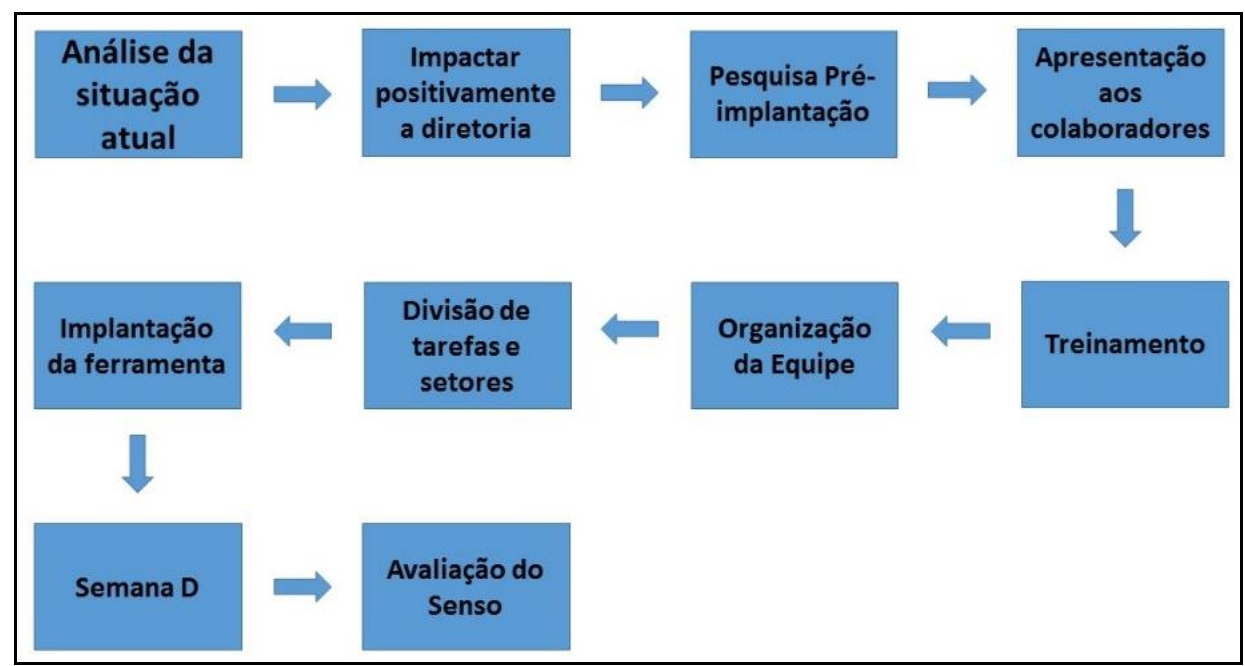

Figura 03 - MAPA DO PROCESSO

Fonte: Própria

Referente ao estudo, o mesmo foi realizado entre os meses de Agosto e Dezembro de 2018. Iniciando-se, primeiramente, levantamentos teóricos, com o intuito de entender a metodologia dos 5 sensos, a fim de proporcionar melhor aproveitamento local desta teoria.

Para identificação de tudo que deveria ser melhorado dentro do local de trabalho, foi necessário uma analise critica levantando questionamento de funcionários e clientes. Com isso, o fluxograma ofereceu direcionamento de cada passo que deveria ser dado, a fim de não deixar que o projeto se perdesse pelo caminho ou acabasse tomando alguma decisão errada.

\section{ESTUDO DE CASO}

\subsection{A EMPRESA}

Fundada em 2012 por Franklin de Oliveira, o Centro Automotivo NEWCAR oferece serviços e produtos para todos os amantes de veículos automotores. Como sócio fundador, Franklin acredita que o diferencial em seus serviços e produtos é a qualidade em volta deles. Desde a sua fundação, o NEWCAR é muito bem visto por seus clientes e carrega uma reputação de peso no bairro Jardim Catarina, localizado na cidade de São Gonçalo no Rio de Janeiro. Comprometimento e clareza foram as palavras utilizadas por ele para definir seu serviço.

\subsection{ANÁLISES E DISCUSSÕES}

No desenvolvimento do projeto, que teve seu início no dia 10/08/2018, os 5 sensos foram explicados e propostos, fundamentados com os conceitos aprendidos durante a revisão bibliográfica e em conjunto com o cenário da empresa. Observou-se que a falta de material era constante no estabelecimento. Isto ocorria devido à dificuldade de seus colaboradores de identificar quais os produtos estavam acabando dentro do estoque. Havia uma ruptura muito 
grande de produtos por falta de fiscalização e organização, e o desperdício contínuo de materiais que não eram guardados de forma correta e acabavam tornando-se inutilizáveis.

Com a aplicação do senso de utilização (Seiri), foi possível a identificação de materiais, equipamentos e até mesmo informações importantes para realização dos serviços ali prestados, como apresenta o quadro 01.

\begin{tabular}{|c|c|c|c|}
\hline № & Verificação & Sim & Não \\
\hline 1 & Ferramentas no devido lugar? & & \\
\hline 2 & Materiais não utilizados foram descartados? & & \\
\hline 3 & Cliente cadastrado no sistema? & & \\
\hline 4 & Os colaboradores realizam a higiene local? & & \\
\hline 5 & Espaço produtivo possui área para descarte? & & \\
\hline 6 & Utensilios separados por setor? & & \\
\hline 7 & $\begin{array}{c}\text { Estoque de materia prima e produtos } \\
\text { acabados devidamente organizado? }\end{array}$ & & \\
\hline 8 & Kit de segurança devidamente instalado? & & \\
\hline 9 & $\begin{array}{r}\text { Todos os equipamentos de campo são } \\
\text { utilizados diariamente? }\end{array}$ & & \\
\hline
\end{tabular}

Quadro 01- Lista de verificação do senso de utilização

Após o estudo do quadro 01, foi possível entender que a empresa não tinha déficit na organização, isto é, o problema principal não era a organização local. Com isso, foi possível aplicar o segundo senso, ordenação/seiton, em que a análise se tornou mais específica para localização dos equipamentos, materiais e informações. O quadro 02 apresenta itens que foram procurados e o resultado que foi obtido.

\begin{tabular}{|c|c|c|c|}
\hline № & Verificação & Sim & Não \\
\hline 10 & Equipamentos identificados? & & \\
\hline 11 & Quadro de aviso atualizado? & & \\
\hline 12 & Facilidade de acesso a objetos ou & & \\
\hline 13 & informações? & & \\
\hline 14 & Sinalização das áres de trabalho por setor? & & \\
\hline 15 & Pastas e documentos etiquetados? & & \\
\hline 16 & Ferramentas organizadas? & & \\
\hline \multicolumn{4}{|c|}{ Quadro 2 - Lista de Verificação para o senso de ordenação } \\
\hline
\end{tabular}

Do estudo do quadro 02, pode-se identificar a ausência de preocupação com o tempo do serviço que será prestado. Visto que o fato de as ferramentas não estarem organizadas, dificulta o acesso a elas e assim compromete a velocidade da execução do serviço. A falta do quadro atualizado é prejudicial, pois deixa os funcionários a mercê de informações que possam vir a ser úteis ao decorrer do dia.

Com relação ao terceiro senso, a limpeza/Seisou, foi possível analisar as condições no qual o local de trabalho, equipamentos e materiais se encontravam. $\mathrm{O}$ quadro 03 apresenta os itens que foram estudados e o resultado que foi obtido. 


\begin{tabular}{|c|c|c|c|}
\hline № & Verificação & Sim & Não \\
\hline 17 & Local para higienização das mãos? & & \\
\hline 18 & Aréa de trabalho com boa iluminação? & & \\
\hline 19 & Os uniformes encontram-se limpos? & & \\
\hline 20 & Ações de limpeza são realizadas sempre? & & \\
\hline 21 & Janelas, paredes, teto e chão encontram-se & & \\
\hline \multicolumn{3}{|c|}{ limpos? } & \\
\hline
\end{tabular}

Pela analise do quadro 03, pode-se concluir que a higienização local não é um ponto forte e nem prioridade. Foram encontradas sujeiras e insetos no ambiente de trabalho por causa de resíduos não retirados de forma correta. Os funcionários não utilizam uniformes apresentáveis, e nem fazem uso de materiais de segurança exigidos pela norma de segurança no trabalho. $\mathrm{O}$ ambiente possui uma boa iluminação e ventilação, o que facilita o trabalho dos colaboradores.

Partindo para o quarto senso, saúde/seiketsu, foi estudado itens que pudessem oferecer riscos a saúde dos funcionários ou clientes. O quadro 04 apresenta os itens que foram estudados e o resultado que foi obtido.

\begin{tabular}{|c|c|c|c|}
\hline $\mathrm{N}^{\mathbf{o}}$ & Verificação & Sim & Não \\
\hline 22 & $\begin{array}{c}\text { Instruções de limpeza para uso de } \\
\text { material coletivo }\end{array}$ & & \\
\hline 23 & Material de Primeiros Socorros & & \\
\hline 24 & $\begin{array}{c}\text { Vistoria da Validade dos Produtos, como } \\
\text { tintas e afins? }\end{array}$ & & \\
\hline 25 & $\begin{array}{c}\text { Estoque segue layout minizando esforço } \\
\text { e transposte de materias pesados? }\end{array}$ & & \\
\hline 26 & Saída de Emergência de Fácil Acesso? & & \\
\hline
\end{tabular}

Quadro 04 - Lista de Verificação para o senso de saúde

De acordo com o quadro 04, o espaço do estoque segue atendendo as normas (NR12) previstas na Associação Brasileira de Normas Técnicas, estando devidamente sinalizado, de fácil acesso a todos os colaboradores, e com isso oferecendo segurança para possam realizar os movimentos de forma correta dentro do local. A falta de higiene local, que já havia sido citada no senso de saúde, permanece nítida. Os colaboradores não gozam de instruções para realizar a limpeza dos materiais, e com isso não tem o habito de higienizalos após o uso, oferecendo então riscos para toda a comunidade.

Após explicar os quatro primeiros sensos, foi a vez de colocar em prática o senso de autodisciplina. Responsável pelo monitoramento contínuo dos primeiros sensos, o shitsuke como é conhecido, garante que a realização da metodologia 5 s seja um ciclo dentro da empresa, e com isso é possível verificar o cumprimento das etapas anteriores. Vejamos o quadro 05 dos itens estudados e analisados. 


\begin{tabular}{|c|l|c|c|}
\hline № & \multicolumn{1}{|c|}{ Verificação } & Sim & Não \\
\hline 27 & $\begin{array}{l}\text { Idetificação dos produtos } \\
\text { encontra-se em bom estado? }\end{array}$ & & \\
\hline 28 & $\begin{array}{l}\text { A limpeza do local de trabalho } \\
\text { vem sendo feita? }\end{array}$ & & \\
\hline 29 & $\begin{array}{l}\text { Ambiente para recepção do } \\
\text { cliente encontra-se em bom } \\
\text { estado? }\end{array}$ & $\begin{array}{l}\text { Estoque segue com identificação } \\
\text { de todos os produtos e } \\
\text { separados por prioridades de } \\
\text { uso? }\end{array}$ & $\begin{array}{l}\text { Funcionários cuidam dos } \\
\text { materiais de sua } \\
\text { responsabilidade? }\end{array}$ \\
\hline \multicolumn{3}{|c|}{ Quadro 05 - Lista de Verificação para o senso de autodisciplina }
\end{tabular}

Após a finalização da vistoria com base no senso de autodisciplina, constatou-se que a limpeza do local de trabalho para recepção dos clientes encontra-se inapropriada. Os materiais de uso coletivo e pessoal seguem sendo higienizados após a utilização, assim como a identificação dos produtos e objetos estão sendo feitas de forma correta e encontram-se em bom estado.

\subsection{RESULTADOS OBTIDOS}

Depois da implantação dos quadros no ambiente de atuação dos mecânicos ficou mais fácil de manter a organização dentro do centro automotivo. Conforme apresenta o gráfico 01 , o tempo para identificação do serviço a ser prestado reduziu em $20 \%$ com relação ao tempo do atendimento sem a metodologia $5 \mathrm{~s}$ implantada.

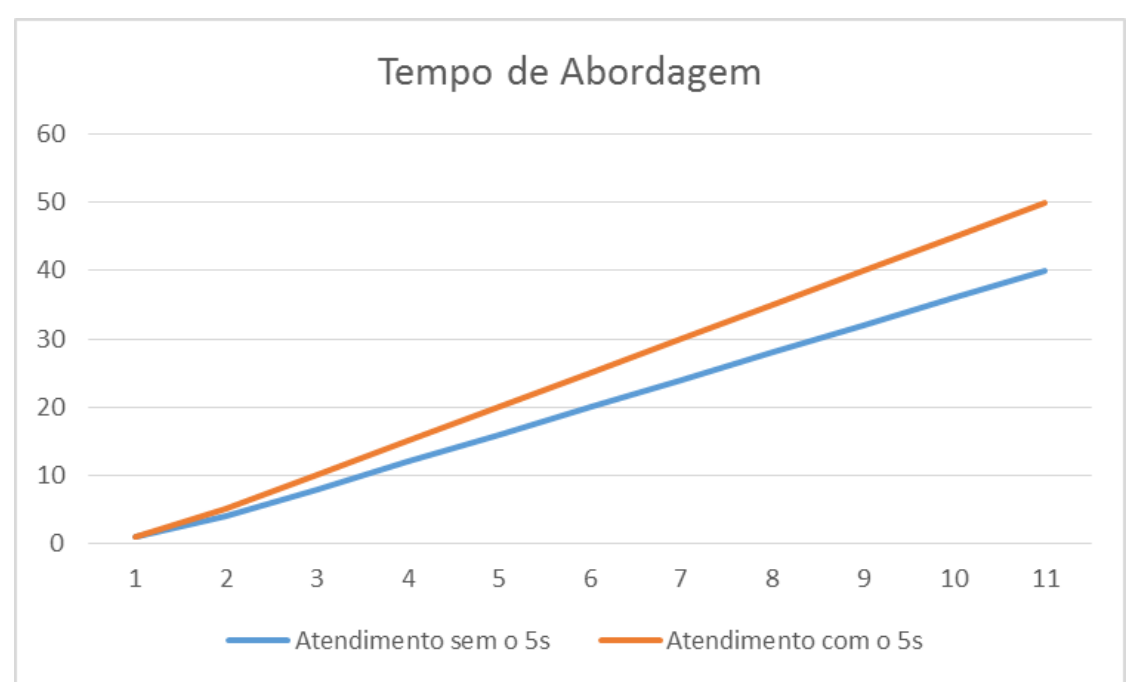

Grafico 01 - Tempo de atendimento antes e após a implantação da metodologia 5 s

Foi notado também a satisfação dos clientes ao encontrar um ambiente agradável, limpo e de fácil acesso a todos os setores. Com isso, a busca pelo serviço prestado no centro 
automotivo aumentou em $15 \%$ com relação aos meses anteriores como apresenta o gráfico 02 .

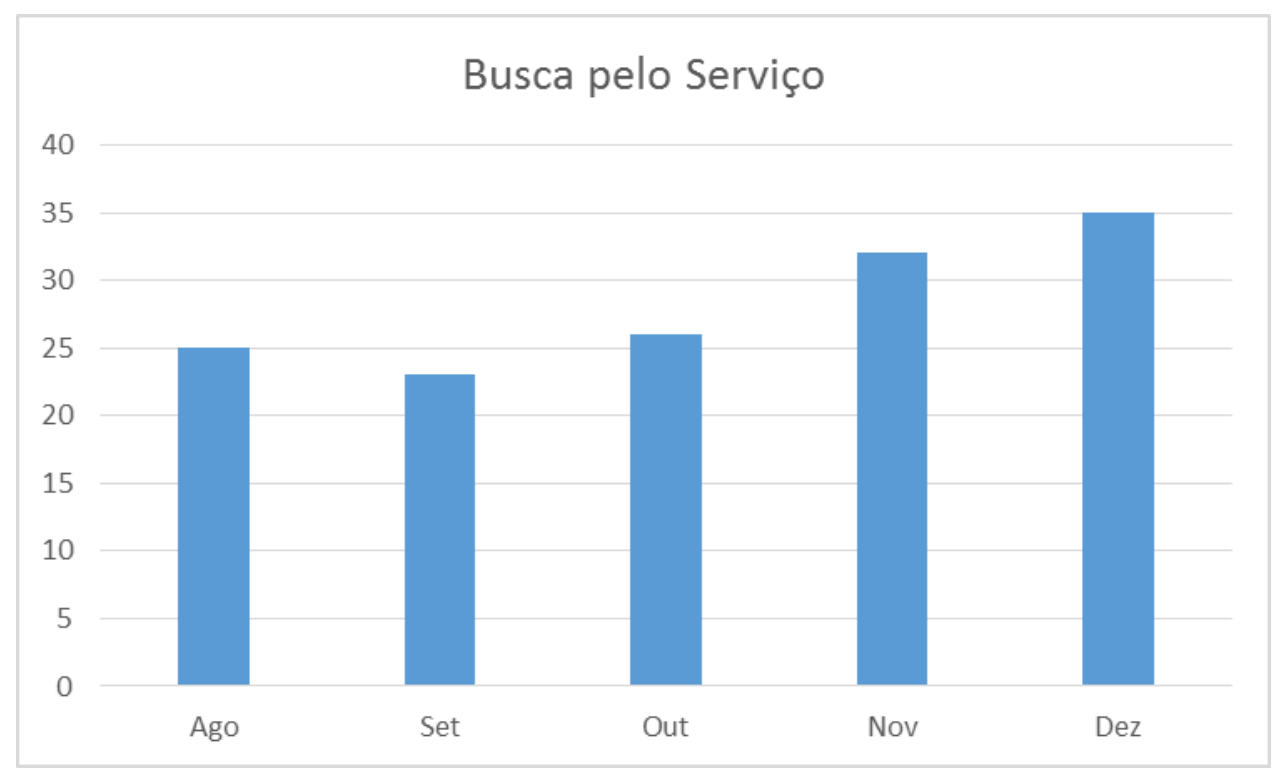

Grafico 02 - Número de atendimento antes e depois implantação a partir do mês Outubro

Com os resultados obtidos após a pesquisa pode se concluir que tanto os clientes quanto os colaboradores aprovaram a metodologia por ser tratar de uma maneira eficiente e de fácil aprendizado. Com relação ao responsável pelo centro automotivo a satisfação também se mostrou presente e o mesmo se comprometeu a garantir que os 5 sensos se tornassem presentes no dia a dia da oficina.

\section{CONSIDERAÇÕES FINAIS}

O presente trabalho resultou em avaliações e melhorias propostas por meio da aplicação da metodologia dos 5 sensos de qualidade, conhecidos como $5 \mathrm{~s}$, no Centro Automotivo NEWCAR. Notou-se que a empresa é composta por colaboradores qualificados, oferecendo um serviço de qualidade aos clientes, a cooperação do responsável pela empresa em melhorar a qualidade de seus serviços e também de oferecer um bom espaço para atuação de seus funcionários, foi muito importante na elaboração e execução da pesquisa.

Todo conhecimento teórico abordado na parte de fundamentos foi crucial para elaboração do conteúdo que seria executado. Com isso ao utilizar as ferramentas propostas, nota-se a facilidade de aplicabilidade dos 5 sensos dentro da oficina, direcionando todos os tipos comprometimento, seja ele individual ou coletivo.

O principal objetivo do trabalho foi atingido, trazendo para o campo de trabalho uma abordagem sistêmica, de fácil entendimento e aplicação, e resultados satisfatórios para toda comunidade.

Portanto, pode-se concluir que as metodologias de gestão pesquisadas são dotadas de boa aplicabilidade dentro do campo selecionado, o que revela atender os objetivos iniciais propostos pelo trabalho. 


\section{REFERÊNCIAS BIBLIOGRÁFICAS}

ALVES, Graciele; BRISOT, Valério. PROGRAMA 5S QUALIDADE TOTAL NAS EMPRESAS. 1a ed. São Paulo. Viena, 2014.

CARVALHO, Pedro Carlos. O PROGRAMA 5S E A QUALIDADE TOTAL. $6^{\circ} \mathrm{ed}$; Campinas: Alínea, 2018.

CHIAVENATO, Idalberto. ADMINISTRAÇÃO DE RECURSOS HUMANOS. São Paulo: Atlas, 2005.

FILHO, Geraldo Vieira. GESTÃO DA QUALIDADE TOTAL: UMA ABORDAGEM PRÁTICA. Campinas. Alínea. 2017.

ISHIKAWA, Kaoru. CONTROLE DE QUALIDADE TOTAL. Rio de Janeiro: Elsevier, 1997.

VERGARA, S.C. Métodos de pesquisa em administração. $6^{\circ}$ ed. São Paulo: Atlas, 2015.

CARVALHO, P. C. O Programa 5S e a Qualidade Total. 6. ed. Campinas: Alínea, 2018. 